\title{
'GP2' - An Energy Resolved Neutron Imaging Detector using a Gd Coated CMOS Sensor
}

\author{
D. E. Pooley, J. W. L. Lee, M. Brouard, R. Farrow, J. J. John, W. Kockelmann, R. B Nickerson, \\ N. J. Rhodes, E. M. Schooneveld, I. Sedgwick, R. Turchetta, C. Vallance
}

\begin{abstract}
This paper documents the R\&D undertaken jointly by the ISIS Neutron Detector Group and the Oxford University PImMS collaboration. The aim of this project was to develop a high resolution, energy resolved, neutron imaging detector named GP2. This conference record introduces the GP2 detector and lists its key physical properties; however the emphasis here will be on the earlier proof-of-principle work performed with both Gadolinium thin films and thick rolled sheets and using the prototype PImMS1 sensor and the larger PImMS2 sensor.
\end{abstract}

\section{INTRODUCTION}

$\mathrm{T}$ $\mathrm{HE}$ increased interest in energy-resolved neutron radiography (ERNR), often shortened to 'neutron imaging', has driven the construction of new dedicated beamlines at spallation sources worldwide. At ISIS (UK) the IMAT [1] beamline specified day-one imaging capability for $2015 / 6$, prioritising this over large area diffraction detector coverage. This paper reports on a new type of neutron detector, named the GP2 detector, which was developed to facilitate the proposed science program. A comprehensive description of the optimised GP2 detector will be published separately [2]. This conference record highlights the earlier proof-of-principle work; a small part of the last three years of R\&D. It demonstrates the feasibility of combining the PImMS CMOS sensor with Gadolinium thin films neutron converters.

\section{DETECTOR INTRODUCTION}

The GP2 detector utilises the PImMS CMOS sensor, so named as it was developed for Particle Imaging Mass Spectrometry, a physical chemistry technique [3]. The sensor

Manuscript received May 5, 2014. PImMS collaboration contact details can be found at pimms.chem.ox.ac.uk.

D. E. Pooley, N. J. Rhodes, E. M. Schooneveld, R. Turchetta, I. Sedgwick and W. Kockelmann are with STFC, Rutherford Appleton Laboratory, Harwell Oxford, Didcot, OX11 0QX, UK, (e-mail author daniel.pooley@stfc.ac.uk).

J. W. L. Lee and C. Vallance are with Department of Chemistry, Chemistry Research Laboratory, 12 Mansfield Rd, University of Oxford, Oxford, UK, (e-mail: jason.lee@ chem.ox.ac.uk).

R. Farrow is with the Campus and Technology Hub, Daresbury Laboratory, Cheshire, UK,. (e-mail: richard.farrow@stfc.ac.uk).

J. J. John and R. B Nickerson are with Department of Physics, Denys Wilkinson Building, Keble Rd, University of Oxford, Oxford, UK, (e-mail: j.john1@physics.ox.ac.uk).

M. Brouard is with the Department of Chemistry, Physical and Theoretical Chemistry Laboratory, South Parks Road, University of Oxford, Oxford, UK (e-mail: mark.brouard@chem.ox.ac.uk). records the time and the spatial position of a detected neutron, generating 'event-mode' data. The PImMS sensor has been made neutron sensitive by sputtering gadolinium on the sensor, meaning the sensor directly detects the generated conversion electrons. The sensor is driven and read out by a compact camera assembly, Fig 1.

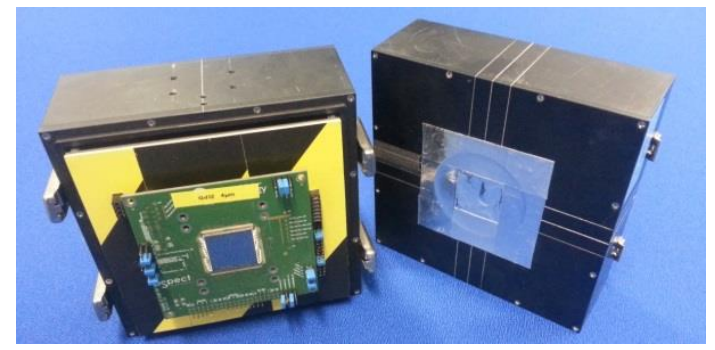

Fig 1. The GP2 detector. Protective cover [RIGHT] removed to reveal the PImMS sensor [LEFT]. The active area is $22.6 \mathrm{~mm} \times 22.6 \mathrm{~mm}$, with the complete detector measuring $15 \mathrm{~cm} \times 15 \mathrm{~cm} \times 11 \mathrm{~cm}$,

\section{DETECTOR SPECIFICATION}

The active area of GP2 is $22.6 \mathrm{~mm} \times 22.6 \mathrm{~mm}$, with a pixel size of $70 \mu \mathrm{m} \times 70 \mu \mathrm{m}$. Each of the 104976 pixels has four independent 12-bit SRAM registers recording the time of neutron detection. Consequently, each pixel can record four independent hits per frame with 4095 available time increments. The sensitivity of each individual pixel can be adjusted, as can the global discrimination threshold parameters. As such, once correctly set-up there is no requirement for a dark-field correction, which is common practice for CCD based imaging. The smallest time binning available is $\Delta \mathrm{t}=12.5 \mathrm{~ns}$, although a typical bin width at a neutron source is $\Delta \mathrm{t} \sim 1 \mu \mathrm{s}$, giving a $4.1 \mathrm{~ms}$ aperture time using the 4095 time increments. The option to set up timegated measurement windows within each frame is integrated into the camera firmware. The user controls the detector at software level instructing the sensor to sample different parts of a TOF spectrum with different time binnings or to pause recording when there is no data of interest. This means that all 4095 time bins and all four independent registers can be used efficiently. Detailed information on the PImMS CMOS sensor is given in [4].

\section{GADOLINIUM CONVERTER WITH THE PIMMS CMOS}

On capture of a neutron the excited gadolinium nucleus decays to its ground state via the release of gamma rays. These in turn produce conversion electrons, which can generate further radiation such as X-rays and Auger 
electrons. The energy distribution of the decay products for this wide range of channels has been modelled extensively by D. A. Abdushukurov [5] with theoretical detection efficiencies calculated allowing for geometry and the propagation/range of the electrons. For any detector to efficiently utilise natural gadolinium as a conversion material, it has to be sensitive to electrons in the energy range $\sim 29 \mathrm{keV}$ to $\sim 132 \mathrm{keV}$, as $\sim 97 \%$ of all ejected electrons have energies in this range. The first experiments with the PImMS sensor were to demonstrate that enough energy would be deposited into the charge collection diodes, approximately $15 \mu \mathrm{m}$ under the sensor surface, to register a neutron hit. This depth is comparable to the maximum range of the low energy electrons generated. It also had to be demonstrated that injecting charge across the entire sensor area would not disrupt the CMOS logic or cause unexpected behavior, as the sensor is designed for detection of visible light, not charged particles.

The first experiments (2012) were performed with the smaller PImMS 1 prototype, in which both spatial and temporal resolution were demonstrated. Later experiments used the larger PImMS 2 sensor with gadolinium directly sputtered to the sensor surface. These proof-of-principle experiments are described below.

\section{Spatial Resolution}

To demonstrate coarse spatial resolution a cadmium mask was used with a $1 \mathrm{~mm}$ hole in the center. For this experiment a $4 \mu \mathrm{m}$ film of gadolinium was pressed against the surface of the sensor. Fig 2 shows the TOF spectrum as measured with the whole sensor and also indicates the two integration ranges used to make the images. The images demonstrate that the spatial resolution is much better than $1 \mathrm{~mm}$. The pronounced peak in TOF is due to the combination of the gadolinium cross section increasing as a function of energy and the cadmium becoming 'opaque' later in TOF. The PImMS detector first 'sees' electrons from the square of gadolinium on the sensor surface and then the cadmium mask as it absorbs colder neutrons.

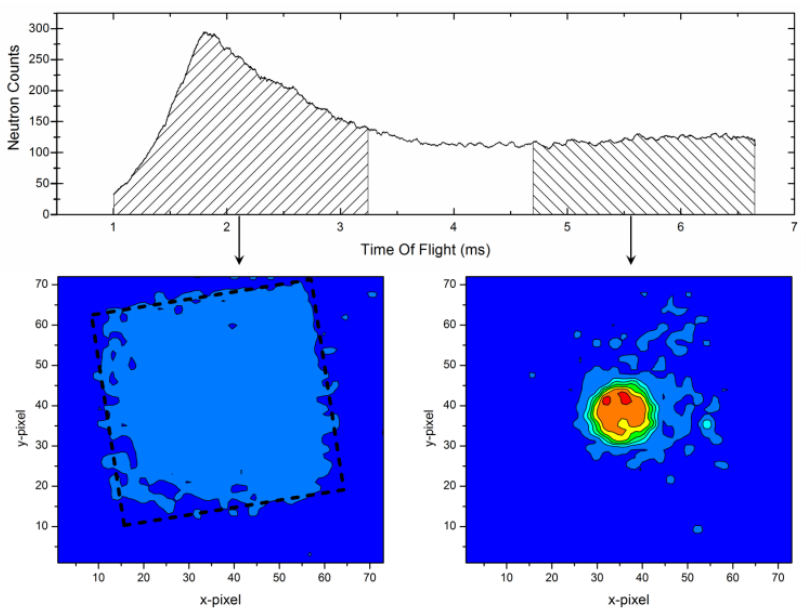

Fig 2. [TOP] TOF spectrum measured, integrated over the whole sensor area. [LEFT] The spatial image obtained for early TOF neutrons showing the gadolinium square. [RIGHT] the spatial image obtained for late TOF showing the $1 \mathrm{~mm}$ aperture in the cadmium mask. One pixel is $70 \mu \mathrm{m}$.

\section{TEMPORAL RESOLUTION}

This experiment was performed with the GP2 detector using the larger PImMS 2 sensor and a $4 \mu \mathrm{m}$ gadolinium thin film directly sputtered onto the surface of the sensor. There was no loss of functionality by sputtering a metallic layer onto the sensor itself.

To demonstrate high temporal resolution, Bragg edges were measured from a $10 \mathrm{~mm}$ thick copper powder sample. Bragg edges from a strain-free powder sample are very sharp features in the TOF spectrum and this must be clearly resolved in order to gain maximum information about the sample. The PImMS sensor can measure at $12.5 \mathrm{~ns}$ intervals, which is substantially faster than the sampling rate required to gain strain information from a Bragg edge. This is confirmed by the clearly resolved copper Bragg edges seen in Fig 3, where the detector sampling rate has been reduced to use time bins of $1.125 \mu \mathrm{s}$.

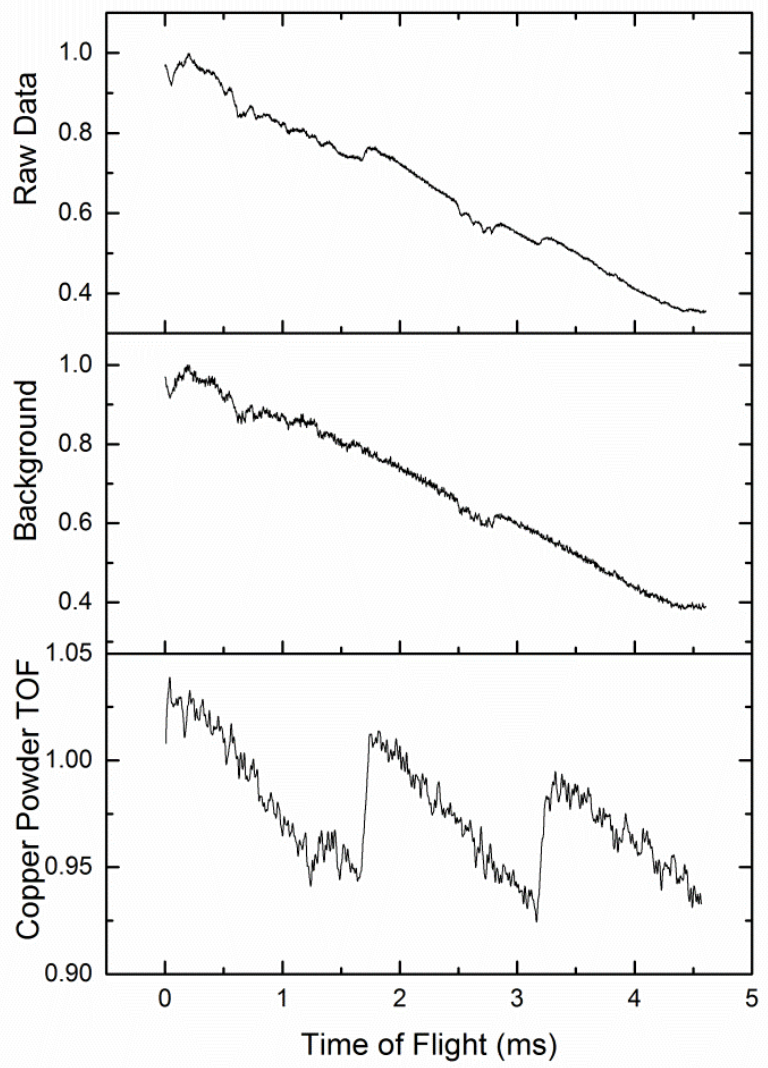

Fig 3. Bragg edge measurement of a copper powder sample. [TOP] The normalized TOF spectrum as recorded. [MIDDLE] The TOF spectrum with the sample out of the beam, used for background subtraction. [BOTTOM] The resulting copper TOF spectrum with clearly resolved Bragg edges.

\section{CONCLUSION AND OUTLOOK}

The results presented document the early proof-of-principle work undertaken at the start of the development of the GP2 detector development program. We have successfully demonstrated that the GP2 detector is sensitive to neutrons and that the sensor is not affected by sputtering thin film gadolinium directly onto the surface of the sensor. This 
means that it can operate as a high temporal and spatial resolution neutron imaging detector without additional mechanics to support the conversion layer. The continued development of GP2 since the proof-of-principle work documented here will be published shortly.

\section{REFERENCES}

[1] W. Kockelmann, S. Y. Zhang, J. F. Kelleher, J. B. Nightingale, G. Burca and J. A. James, "IMAT - A New Imaging and Diffraction Instrument at ISIS," Physics Procedia, vol. 43, pp. 100-110, 2013.

[2] D. E. Pooley, J. W. L. Lee, M. Brouard, R. Farrow, J. J. John, W. Kockelmann, R. B. Nickerson, N. J. Rhodes, E. M. Schooneveld, I. Sedgwick, R. Turchetta and C. Vallance, "Manuscript in preparation," 2016.

[3] C. Vallance, M. Brouard, A. Lauer, C. S. Slater, E. Halford, B. Winter, S. J. King, J. W. L. Lee, D. E. Pooley, I. Sedgwick, R. Turchetta, A. Nomerotski, J. J. John and L. Hill, "Fast sensors for time-of-flight imaging applications," Phys. Chem. Chem. Phys., vol. 16, pp. 383395, 2013.

[4] J. J. John , M. Brouard, A. Clark, J. Crooks , E. Halford, L. Hill, J. J. W. Lee, A. Nomerotski , R. Pisarczyk, I. Sedgwick, C. S. Slater, R. Turchetta, C. Vallance, E. Wilman, B. Winter and W. H. Yuen, "PImMS, a fast event-triggered monolithic pixel detector with storage of multiple timestamps," JINST, vol. 7, p. C08001, 2012.

[5] D. A. Abdushukurov, Gadolinium Foils as Converters of Thermal Neutrons in Detectors of Nuclear Radiation, Nova Science Publishers, 2010. 